\title{
Fluid-Gravity Correspondence under the presence of viscosity
}

\author{
B. González-Fernández* \\ Instituto de Ciencias Nucleares, Universidad Nacional Autónoma de México, \\ A. Postal 70-543, C.P. 04510, México, D.F., México. \\ A. Camachd甲 \\ Departamento de Física, Universidad Autónoma Metropolitana-Iztapalapa \\ Apartado Postal 55-534, C.P. 09340, México, D.F., México.
}

(Dated: November 10, 2018)

\begin{abstract}
The present work addresses the analogy between the speed of sound of a viscous, barotropic, and irrotational fluid and the equation of motion for a non-massive field in a curved manifold. It will be shown that the presence of viscosity implies the introduction, into the equation of motion of the gravitational analogue, of a source term which entails the flow of energy from the non-massive field to the curvature of the spacetime manifold. The stress-energy tensor is also computed and it is found not to be constant, which is consistent with such energy interchange.
\end{abstract}

PACS numbers: 04.50+h, 04.20.Jb, 11.25.Mj, 04.60.Bc

\section{INTRODUCTION}

Analogies have always played a fundamental role in physics and mathematics. Indeed, they define a bridge between two different branches of science. One of the current trends in this direction is the so-called Analogue Gravity, a topic that considers the similarities between physics in a curved spacetime and, usually, condensed matter systems [1]. The movement of light waves in a curved manifold finds a beautiful analogy in the description of sound waves in a moving fluid.

Besides the inherent beauty of analogies, they are also important as they represent a powerful tool that can give us an insight of what might be happening in a system based on phenomena that occur in its correspondent analogue. Of course, at this point, a warning word is a must. Indeed, the existence of an analogy does not imply a complete equivalence between the aforementioned theories. In other words, the main objective of an analogy is to capture a sufficient number of relevant features of the modeled theory.

In particular, the search for analogies between condensed matter and gravitational cases are interesting because they could shed some light upon some major problems in gravity that still remain unsolved. For instance, since condensed matter physics is described resorting to a quantum field theory which can be tested experimentally, the analysis of the analogies between particle physics, particularly the quantum vacuum, and condensed matter could bring light into the physics for trans-Planckian situations [2].

One of the simplest cases involving a moving fluid and an analogue spacetime is provided by the acoustics of the fluid [3]. In this example the existence of a silent hole has

* belinkag@nucleares.unam.mx

† acq@xanum.uam.mx been shown, namely, a region from which sound is unable to escape. This resembles the situation of a black hole in general relativity [4].

The analysis of the speed of sound in a fluid and the possibility of shaping this case in the form of the equation of motion for a non-massive field immersed in a curved spacetime is already an old result [1]. The logical premises in the aforementioned work consider a barotropic, inviscid and irrotational flow. The result is that the equation of motion for the corresponding velocity potential can be cast as that of a minimallycoupled scalar field, without mass, which propagates in a Lorentzian geometry. It has to be underlined that this equation of motion has a null source term. The generalization of this result, namely, the possibility of having a rotational fluid has also already been carried out. Here we may state that, when non-vanishing vorticity is present in a fluid, the analogy with gravitation can be done, but the Unruh acoustic metric [5] does not suffice, i.e., the introduction of Cartan torsion is compulsory [ 6 ].

In the present work, we consider a barotropic and irrotational fluid, but now we allow a non-vanishing viscosity. We will show that the equation of motion for the corresponding velocity potential can be considered as that of a non-massive and minimally coupled field propagating in a $(3+1)$ Lorentzian geometry. In contrast to [1], now a source term appears which is induced by the presence of viscosity. This last feature can be interpreted, physically, as an element that implicates the transfer of energy from the field to the curvature of the manifold.

\section{ANALOGUE GRAVITY AND VISCOSITY}

In the spirit of [1] we now proceed to prove the following. 


\section{Theorem}

Let us consider an irrotational and barotropic fluid, though with a non-vanishing viscosity. The equation of motion for the velocity potential associated to an acoustic perturbation, $\phi_{1}$, is identical to the equation of motion of a minimally coupled non-massive field in a $(3+1)-$ Lorentzian geometry in which a source term is present:

$$
\partial \mu\left[\sqrt{-g} g^{\mu \nu} \partial_{\nu} \phi_{1}\right]=\partial \mu\left[\sqrt{-g} g^{0 \mu} \frac{\nabla^{2} \phi_{1}}{R}\right] .
$$

The source contribution entails an energy flow from the non-massive field to the curvature of the manifold.

\section{Proof}

We start writing down our equations of motion, which in our case are the continuity equation and the NavierStokes equations [10]

$$
\begin{gathered}
\frac{\partial \rho}{\partial t}+\nabla \cdot(\rho \vec{v})=0 \\
\rho \frac{D \vec{v}}{D t}=-\nabla p+\left(\zeta+\frac{1}{3} \eta\right) \nabla(\nabla \cdot \vec{v})+\eta \nabla^{2} \vec{v} .
\end{gathered}
$$

Here $p$ denotes pressure (the existence of the concept of pressure is related to the definition of ideal fluid [7], $\rho$ is the density, $\zeta$ and $\eta$ are viscosity coefficients, $\vec{v}$ is the velocity of the fluid element, and $D / D t=\partial / \partial t+\vec{v} \cdot \nabla$ is the material derivative [7] .

We now impose the condition of vanishing vorticity

$$
\nabla \times \vec{v}=\overrightarrow{0}
$$

Additionally, we require the fluid to be barotropic, i.e., enthalpy has to be a function of the pressure only [1]. Under these condition, and using some vector analysis formulas, we obtain that (3) reduces to

$$
\rho \frac{D \vec{v}}{D t}=-\nabla p+\left(\zeta+\frac{4}{3} \eta\right) \nabla(\nabla \cdot \vec{v}) .
$$

The barotropic behavior of the fluid means there exists a function $h(p)$, called enthalpy, such that

$$
h(p)=\int^{p} \frac{d p^{\prime}}{\rho\left(p^{\prime}\right)},
$$

hence

$$
\frac{1}{\rho} \nabla p=\nabla h(p) .
$$

The final assumption involves the behavior of the coefficient $\mu \equiv\left(\zeta+\frac{4}{3} \eta\right)$, which we assume that changes slower than the remaining parameters.
We now define the concept of velocity potential, $\phi$, which is well-defined due to the condition of vanishing vorticity [7],

$$
\vec{v}=-\nabla \phi
$$

Then our equation of motion can be shaped in the following form

$$
\nabla\left[\partial_{t} \phi-h-\frac{1}{2}(\nabla \phi)^{2}+\frac{\mu}{\rho}\left(\nabla^{2} \phi\right)\right]=0
$$

so that it reads

$$
\partial_{t} \phi-h-\frac{1}{2}(\nabla \phi)^{2}+\frac{\mu}{\rho}\left(\nabla^{2} \phi\right)=0 .
$$

We now proceed to introduce the idea of sound in the context of fluctuations in our physical parameters around the equilibrium state. Keeping the expansions up to linear terms of our small parameter, denoted here by $\alpha$, the thermodynamical variables become, asymptotically, [8],

$$
\begin{gathered}
\rho=\rho_{e}+\alpha \rho_{s}, \\
p=p_{e}+\alpha p_{s}, \\
\phi=\phi_{e}+\alpha \phi_{s}, \\
h(p)=h\left(p_{0}\right)+\alpha \frac{p_{1}}{\rho_{0}}+\ldots
\end{gathered}
$$

Introducing these expansions into the equation of motion we obtain two different equations: one that shows no dependence upon $\alpha$ and another that hinges linearly on $\alpha$. Indeed,

$$
\begin{gathered}
\partial_{t} \phi_{0}-h_{0}-\frac{1}{2}\left(\nabla \phi_{0}\right)^{2}-\frac{\mu}{\rho}\left(\nabla^{2} \phi_{0}\right)=0, \\
\partial_{t} \phi_{1}+\vec{v}_{0} \cdot \nabla \phi_{1}-\frac{p_{1}}{\rho_{0}}-\frac{\mu}{\rho} \nabla^{2} \phi_{1}=0
\end{gathered}
$$

where we have assumed $\nabla \cdot \vec{v}_{0}=0$.

In order to have a consistent system, we must introduce our expansions in the continuity equation (see (2)), to obtain

$$
\begin{gathered}
\frac{\partial \rho_{0}}{\partial t}+\nabla \cdot\left(\rho_{0} \vec{v}_{0}\right)=0 \\
\frac{\partial \rho_{1}}{\partial t}-\nabla \cdot\left(\rho_{0} \nabla \phi_{1}+\phi_{1} \nabla \phi_{0}\right)=0 .
\end{gathered}
$$


Finally, the restriction concerning the barotropic behavior leads to

$$
\rho_{1}=\frac{\partial \rho}{\partial p} p_{1}
$$

Our previous results allow us to mold this last expression in the following form

$$
\rho_{1}=\frac{\rho_{0}}{c^{2}}\left[\partial_{t} \phi_{1}+\vec{v}_{0} \cdot \nabla \phi_{1}-\frac{\mu}{\rho} \nabla^{2} \phi_{1}\right]
$$

Therefore, the equation of motion becomes

$$
\begin{array}{r}
-\frac{\partial}{\partial t}\left\{\frac{\rho_{0}}{c^{2}}\left[\partial_{t} \phi_{1}+\vec{v}_{0} \cdot \nabla \phi_{1}-\frac{\mu}{\rho_{0}} \nabla^{2} \phi_{1}\right]\right\} \\
+\nabla \cdot\left\{\rho_{0} \nabla \phi_{1}-\vec{v}_{0} \frac{\rho_{0}}{c^{2}}\left[\partial_{t} \phi_{1}\right.\right. \\
\left.\left.+\vec{v}_{0} \cdot \nabla \phi_{1}-\frac{\mu}{\rho_{0}} \nabla^{2} \phi_{1}\right]\right\}=0 .
\end{array}
$$

This equation can be cast in the following form

$$
\partial_{\mu}\left[f^{\mu \nu} \partial_{\nu} \phi_{1}\right]=\partial_{\mu}\left[f^{0 \mu} \frac{\mu}{\rho_{0}} \nabla^{2} \phi_{1}\right] .
$$

In this last expression we have that

$$
f^{\mu \nu} \equiv \frac{\rho_{0}}{c^{2}}\left(\begin{array}{ccc}
-1 & \vdots & -v_{0}^{j} \\
\cdots & \cdots \\
-v_{0}^{i} & \vdots & c^{2} \delta^{i j}-v_{0}^{i} v_{0}^{j}
\end{array}\right)
$$

In order to obtain the analogy with the case of a scalar d'Alembertian in a curved space $(\Delta \phi=$ $\left.(1 / \sqrt{-g}) \partial_{\mu}\left(\sqrt{-g} g^{\mu \nu} \partial_{\nu} \phi\right)\right)$ we deduce that our metric is provided by $f^{\mu \nu}=\sqrt{-g} g^{\mu \nu}$, and $\sqrt{-g}=\rho_{0}^{2} / c$. Hence, the equation of motion reads

$$
\begin{aligned}
& \frac{1}{\sqrt{-g}} \partial_{\mu}\left(\sqrt{-g} g^{\mu \nu} \partial_{\nu} \phi_{1}\right)= \\
& \frac{1}{\sqrt{-g}} \partial_{\mu}\left[\sqrt{-g} g^{0 \mu} \frac{\mu}{c^{2}} \nabla^{2} \phi_{1}\right] .
\end{aligned}
$$

\section{STRESS-ENERGY TENSOR}

In order to understand what is happening with energy in this system, we calculate the stress-energy tensor [4].

As usual, the energy density of the fluid element will be encoded in the component $T^{00}$, so that

$$
T^{00}=\rho \varepsilon+\frac{1}{2} \rho v^{2},
$$

where $\varepsilon$ represents the internal energy per unit mass.

Meanwhile, the components $T^{i 0}=T^{0 i}$, expressing the $i$-th component of the momentum, will be

$$
T^{i 0}=T^{0 i}=\rho v_{i}
$$

To find the $T^{i j}$ components of the tensor, corresponding to the flux of the $i$-th component of the momentum through a surface with $x^{j}=$ constant, we compute the rate of change of the $i$-th momentum in time

$$
\frac{\partial}{\partial t}\left(\rho v_{i}\right)=\rho\left(\frac{\partial v_{i}}{\partial t}\right)+\left(\frac{\partial \rho}{\partial t}\right) v_{i}
$$

Substituting the continuity equation (2) and the Navier-Stokes equations (3) in (27), we can rewrite it as

$$
\begin{array}{r}
\frac{\partial}{\partial t}\left(\rho v_{i}\right)=-\frac{\partial}{\partial x_{k}}\left\{\rho v_{i} v_{k}+\left[p-\mu \frac{\partial v_{l}}{\partial x_{l}}\right] \delta_{i j}\right\} \\
\equiv-\frac{\partial}{\partial x_{k}}\left(\Pi_{i k}\right),
\end{array}
$$

where we have conveniently defined the tensor

$$
\Pi_{i k} \equiv \rho v_{i} v_{k}+\left[p-\mu \frac{\partial v_{l}}{\partial x_{l}}\right] \delta_{i j} .
$$

If we integrate (28) over some volume $V$,

$$
\frac{\partial}{\partial t} \int \rho v_{i} d V=\int-\frac{\partial \Pi_{i k}}{\partial x_{k}}=\oint \Pi_{i k} \widehat{n}_{k} d S,
$$

it is easy to see that the left side of (30) is the rate of change of the $i$-th component of the momentum per unit time, so that $\Pi_{i k}$ will be the flux of the $i$-th component of the momentum through a surface with $x^{j}=$ constant, thus

$$
T^{i k}=\Pi^{i k}=\rho v_{i} v_{k}+\left[p-\mu \frac{\partial v_{l}}{\partial x_{l}}\right] \delta_{i j} .
$$

Having all its components, we combine (25), (26) and (31) to form a single matrix representing the stressenergy tensor:

$$
\left(T^{\mu \nu}\right)=\left(\begin{array}{ccc}
\rho \varepsilon+\frac{1}{2} \rho v^{2} & \vdots & \rho v_{i} \\
\cdots & \cdots \\
\rho v_{i} & \vdots & \rho v_{i} v_{k}+\left[p-\mu \frac{\partial v_{l}}{\partial x_{l}}\right] \delta_{i j}
\end{array}\right) .
$$

Finally, we linearize the components of the tensor.

For $T^{00}$, we find

$$
\begin{gathered}
T_{(0)}^{00}=\rho_{0} h_{0}-p_{0}+\frac{1}{2} \rho_{0} v_{0}^{2} \\
T_{(1)}^{00}=\rho_{1} h_{0}+\frac{1}{2}\left(\rho_{1} v_{0}^{2}\right)+\frac{1}{2}\left(\rho_{0} \vec{v}_{0} \cdot \vec{v}_{1}\right),
\end{gathered}
$$

where we have expressed $\varepsilon$ in terms of the enthalpy, using the deffinition of the latter.

In the case of $T^{0 i}$, the result is

$$
\begin{gathered}
T_{(0)}^{0 i} \equiv \rho_{0} v_{0}^{i} \\
T_{(1)}^{0 i} \equiv \rho_{0} v_{1}^{i}+\rho_{1} v_{0}^{i} .
\end{gathered}
$$


At last, for $T^{i j}$ we have

$$
\begin{gathered}
T_{(0)}^{i j} \equiv \rho_{0} v_{0}^{i} v_{0}^{j}+\left(p_{0}-\mu \nabla \cdot \vec{v}_{0}\right) \delta^{i j} \\
T_{(1)}^{i j} \equiv \rho_{1} v_{0}^{i} v_{0}^{j}+\rho_{0}\left(v_{1}^{i} v_{0}^{j}+v_{0}^{i} v_{1}^{j}\right)+\left(p_{1}-\mu \nabla \cdot \vec{v}_{1}\right) \delta^{i j} .
\end{gathered}
$$

It should be noted that, when we make $\rho_{1} \rightarrow 0, \vec{v}_{1} \rightarrow 0$, and $p_{1} \rightarrow 0$, the inviscid case is recovered.

Harking back to our particular case, these aforementioned parameters are not constant. In other words, clearly there is some interchange of energy between the scalar field and the spacetime, as the first transfers part of its energy to the second.

\section{CONCLUSIONS AND DISCUSSION}

Considering the case $\zeta=0$ and $\eta=0$ we recover the usual theorem [1]. Observe that, in our case, the presence of a non-vanishing viscosity appears as a source term for the equation of motion of the non-massive field. Let us now provide a physical interpretation, in the context of (24), for the role of viscosity. Viscous terms provide a mechanism by which momentum transfer becomes relevant in a fluid. Another way to understand the role of viscosity is noting that it provides a mechanism by which macroscopic energy can be turned into internal energy 8]. This last statement allows us to comprehend, at the level of our main result, this issue. Indeed, the aforementioned flow of energy implies that, for our last equation, we may assert that our parameter $\mu$, which contains the information concerning viscosity, is responsible for the flow of energy from our field $\phi_{1}$ into the curvature of the manifold, a fact already shown in the previous section.

It is readily seen that the left hand side of our main result, (24), is associated to a non-massive and spinless field, such as a non-massive boson with vanishing spin. In other words, our equation would provide the dynamics of a particle with these features moving in a curved spacetime, assuming also, of course, a coupling with the curvature of the kind here introduced. It has to be underlined that the flow of energy from a system into the curvature of a manifold does not, necessarily, imply a breakdown of the postulates of GR. For instance, in GR a system radiating gravitational waves loses its energy, which becomes "ripples" of spacetime, i.e., ripples of the curvature.

\section{ACKNOWLEDGMENTS}

B. González-Fernández thanks CONACYT for the grant received.
[1] C. Barceló, S. Liberati, and M. Visser Liv. Rev.. 83, 2876-2879 (2012).

[2] G. E. Volovik, The Universe in a Helium Droplet, Oxford Science Publications, Oxford (2002).

[3] M. Visser, Clas. Quant. Grav. 15, 1767-1791 (1998).

[4] C. Misner, K. Thorne, and J. A. Wheeler, Gravitation, W. H. Freeman, San Francisco, (1973).

[5] W. Unruh, Phys. Rev. Lett. , 1351-1353 (1981).

[6] L. C. Garcia de Andrade, Phys. Rev. D70, 064004 (2004); Phys. Lett. A339, 188-193 (2005); Phys. Lett.
A346, 327-329 (2005).

[7] A. L. Chorin and J. E. Marsden, A Mathematical Introduction to Fluid Mechanics, Springer-Verlag, New York, (2000).

[8] M. H. Holmes, Introduction to Perturbation Methods, Springer-Verlag, New York, (1998).

[9] T. Misner and Wheeler, Gravitation, W. H. Freeman and Company, 1973.

[10] L. D. Landau and E. M. Lifshitz, Fluid Mechanics, Pergamon Press, 1987. 